\title{
Variation in Influenza B Virus Epidemiology by Lineage, China
}

\section{Juan Yang, ${ }^{1,2}$ Yiu Chung Lau, ${ }^{1}$ Peng Wu, ${ }^{1}$ Luzhao Feng, Xiling Wang, Tao Chen, Sheikh T. Ali, Zhibin Peng, Vicky J. Fang, Juanjuan Zhang, Yangni He, Eric H.Y. Lau, Ying Qin, Jing Yang, Jiandong Zheng, Hui Jiang, Hongjie Yu, ${ }^{1,2}$ Benjamin J. Cowling}

We used national sentinel surveillance data in China for 2005-2016 to examine the lineage-specific epidemiology of influenza B. Influenza B viruses circulated every year with relatively lower activity than influenza $A$. B/Yamagata was more frequently detected in adults than in children.

I nfluenza B virus, first identified in 1940 (1), is associated with considerable hospital admissions and deaths worldwide every year (2). During the early 1980s, influenza B viruses split into 2 lineages, termed B/ Victoria and B/Yamagata (3). These 2 lineages showed distinct antigenicity and transmission dynamics (4) and have co-circulated during each influenza season since 2001 (2). Relatively less attention has been given to influenza B virus epidemiology than to influenza A epidemiology (2) because influenza B virus spreads almost exclusively in humans and does not pose a pandemic threat (5).

Several recent reports have highlighted potential differences in the epidemiology of B/Victoria and B/ Yamagata lineage viruses, including younger average ages of persons with $\mathrm{B} /$ Victoria virus infection $(4,6,7)$ and greater transmissibility of B/Victoria viruses $(4,6)$. Our study aimed to describe epidemiologic patterns of influenza B virus activity in China and to identify and compare the seasonality and age distribution of persons with medically attended influenza $\mathrm{B} /$ Victoria and $\mathrm{B} / \mathrm{Ya}-$ magata virus infections.

\footnotetext{
Author affiliations: Chinese Center for Disease Control and Prevention, Beijing, China (Juan Yang, L. Feng, T. Chen, Z. Peng, Y. Qin, Jing Yang, J. Zheng, H. Jiang, H. Yu); Fudan University School of Public Health, Key Laboratory of Public Health Safety, Ministry of Education, Shanghai, China (Juan Yang, X. Wang, J. Zhang, Y. He, H. Yu); The University of Hong Kong, Hong Kong, China (Y.C. Lau, P. Wu, S.T. Ali, V.J. Fang, E.H.Y. Lau, B.J. Cowling)
}

DOI: https://doi.org/10.3201/eid2408.180063

\section{The Study}

The Chinese Center for Disease Control and Prevention coordinated influenza surveillance in sentinel clinics during October 2005-March 2016. Sentinel hospitals in provinces in southern China conducted year-round surveillance; in northern China (except for Liaoning, Gansu, and Tianjin provinces, where year-round surveillance was conducted), surveillance was suspended from April to September before 2009 because influenza has low activity in summer in these temperate areas of China (8). Sentinel surveillance was then expanded from 193 to 554 hospitals conducting year-round surveillance in all provinces since 2009. Sentinel hospitals reported the number of outpatients and the number of outpatients with influenza-like illness symptoms on a daily basis. Respiratory specimens collected from a subset of outpatients with influenza-like illness were tested for influenza viruses. Each sentinel hospital in northern China was required to collect 10-15 samples per week during October-March and 5-15 samples per month during April-September for virus testing, and the hospitals in southern China tested 5-15 samples per week throughout the year. Most laboratories had adopted real-time PCR for lineage identification since 2009; some laboratories still use virus culture followed by hemagglutination inhibition test (online Technical Appendix, https://wwwnc.cdc. gov/EID/article/24/8/18-0063-Techapp1.pdf). Individual data on age, sex, and date of specimen collection also were reported for all selected patients for virus testing (online Technical Appendix). Because national influenza sentinel surveillance was part of a routine public health investigation, the study was exempt from institutional review board assessment, and all data were delinked from identifiable personal information.

We used a proxy measure of influenza activity in the communities served by the sentinel locations because it was previously indicated to be a good correlate of the incidence rates of influenza virus infection in the community (9). The proxy was calculated as the product of the weekly rates for influenza-like illness consultation and the proportion of sentinel specimens testing positive for each lineage in the same week. The age-specific proportions of sentinel

\footnotetext{
${ }^{1}$ These authors contributed equally to this article.

${ }^{2}$ Current affiliation: Fudan University School of Public Health, Key Laboratory of Public Health Safety, Ministry of Education, Shanghai, China.
} 
specimens testing positive for influenza $\mathrm{B}$ virus by lineage were derived as the proportion of sentinel specimens testing positive for each lineage (numerator) among the outpatients recruited for specimen collection (denominator) by exact year of age.

We found that influenza B/Victoria and B/Yamagata lineages circulated every year in mainland China during 2005-2016 and were mostly active during the winterspring seasons (Figure 1). Influenza B virus activity was generally less intense than influenza A activity and less apparent during the 2005-06, 2010-11, and, particularly, 2012-13 seasons (Figure 1; online Technical Appendix Figure 1).
Influenza B/Victoria activity increased in every season before and during the first wave of infections with influenza A(H1N1)pdm09 virus in China in late 2009, whereas substantial virus detections were only seen in the early 2011 12 and 2015-16 seasons during the postpandemic period. $\mathrm{B} /$ Yamagata lineage led to 3 major epidemics during the 2007-08, 2013-14, and 2014-15 seasons (online Technical Appendix Figure 1). These major epidemics were associated with prolonged influenza activity, particularly during summer periods and in provinces and municipalities with lower latitude, which occurred during 2008-2011 for B/ Victoria lineage and during the 2007-08 and 2014-15 seasons for B/Yamagata lineage (Figure 1).
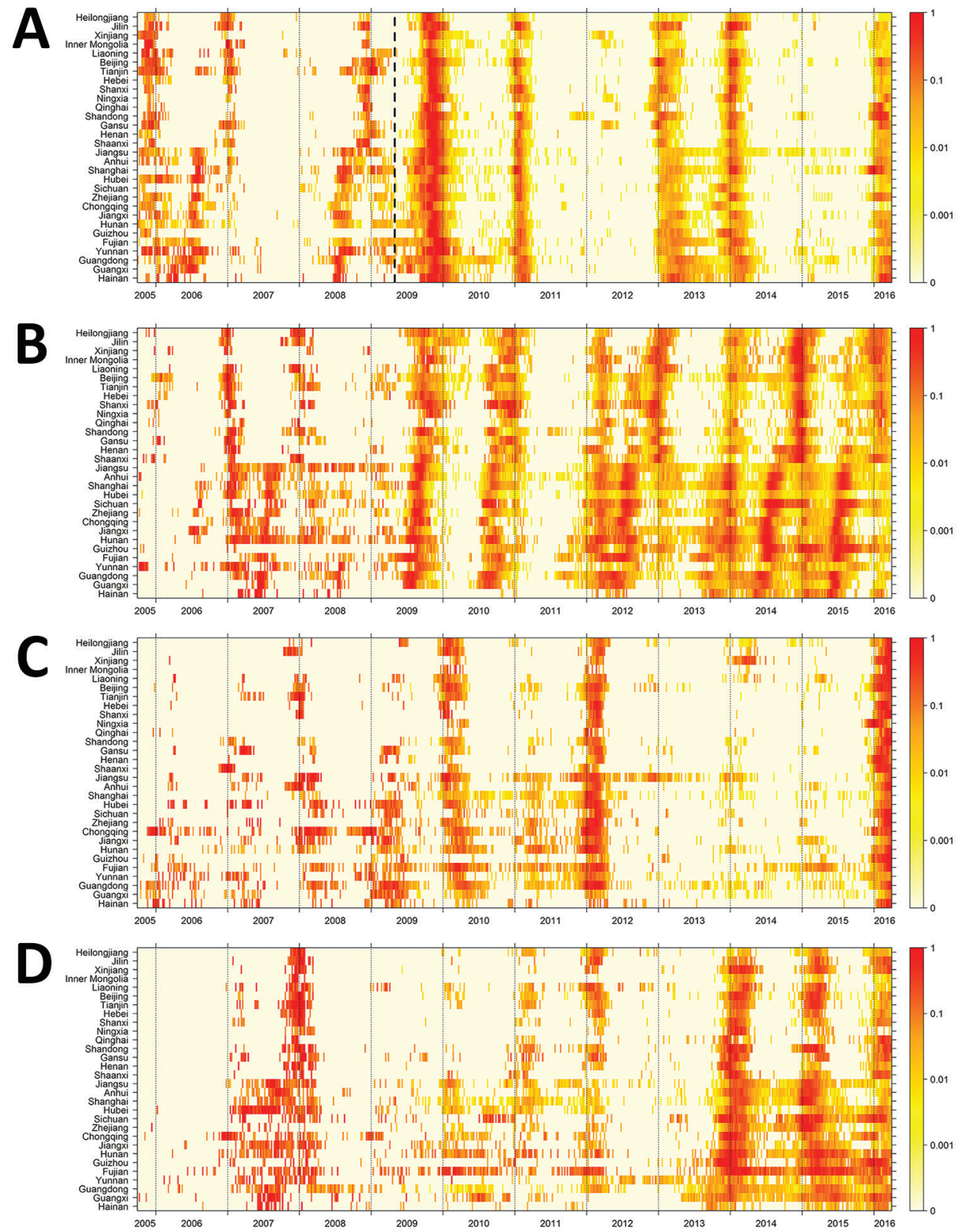

Figure 1. Heatmap of influenza virus activity by lineage in 30 provinces and municipalities (sorted by latitude), China, October 2005-March 2016. A) Influenza A(H1N1); B) influenza $\mathrm{A}(\mathrm{H} 3 \mathrm{~N} 2) ; \mathrm{C})$ influenza B/Victoria lineage; D) influenza B/Yamagata lineage. Map is based on $2,498,735$ specimens collected from the sentinel hospitals. Normalized virus activity is shown for each province and municipality as the product of the weekly proportion of influenzalike illness consultations and the weekly proportion of sentinel specimens testing positive for influenza viruses divided by the maximum virus activity in the province or municipality throughout the study period to give a rescaled proxy with values between 0 (no activity) and 1 (highest activity in that province). The dashed line in panel $A$ indicates the start of the H1N1 pandemic in 2009. 
Children 5-15 years of age had the highest detection rates among all age groups for both lineages. The rates of detection of $\mathrm{B} / \mathrm{Victoria}$ lineage viruses decreased with age after peaking at 10 years of age, and the rates of $\mathrm{B} /$ Yamagata lineage virus infections generally increased among persons $>25$ years of age to a second peak in older adults (Figure 2, panel A). The patterns differed somewhat across provinces and municipalities without systematic variation by latitude (online Technical Appendix Figure 2). In comparison, influenza $A(\mathrm{H} 1 \mathrm{~N} 1)$ showed an age pattern similar to that for $\mathrm{B} /$ Victoria but with a later peak, at 10-20 years of age; however, influenza A(H3N2) indicated largely comparable virus detections across different age groups (Figure 2, panel B).

\section{Conclusions}

Our study showed that influenza B virus generally was relatively less active than influenza A virus (Figure 1; online Technical Appendix Figure 1). Influenza B/Yamagata caused fewer epidemics than B/Victoria during the study period, largely consistent with findings from a study using sentinel surveillance data from multiple countries (10). The alternating predominance of the $\mathrm{B} /$ Victoria and $\mathrm{B} / \mathrm{Ya}$ magata lineages, especially after 2009 , and the low influenza B virus activity in China during the $2012-13$ season might reflect the complex interactions between population immunity and virus evolution of influenza B lineages (11).

Our study suggested a potential difference in the age patterns of persons infected with $\mathrm{B} /$ Yamagata and

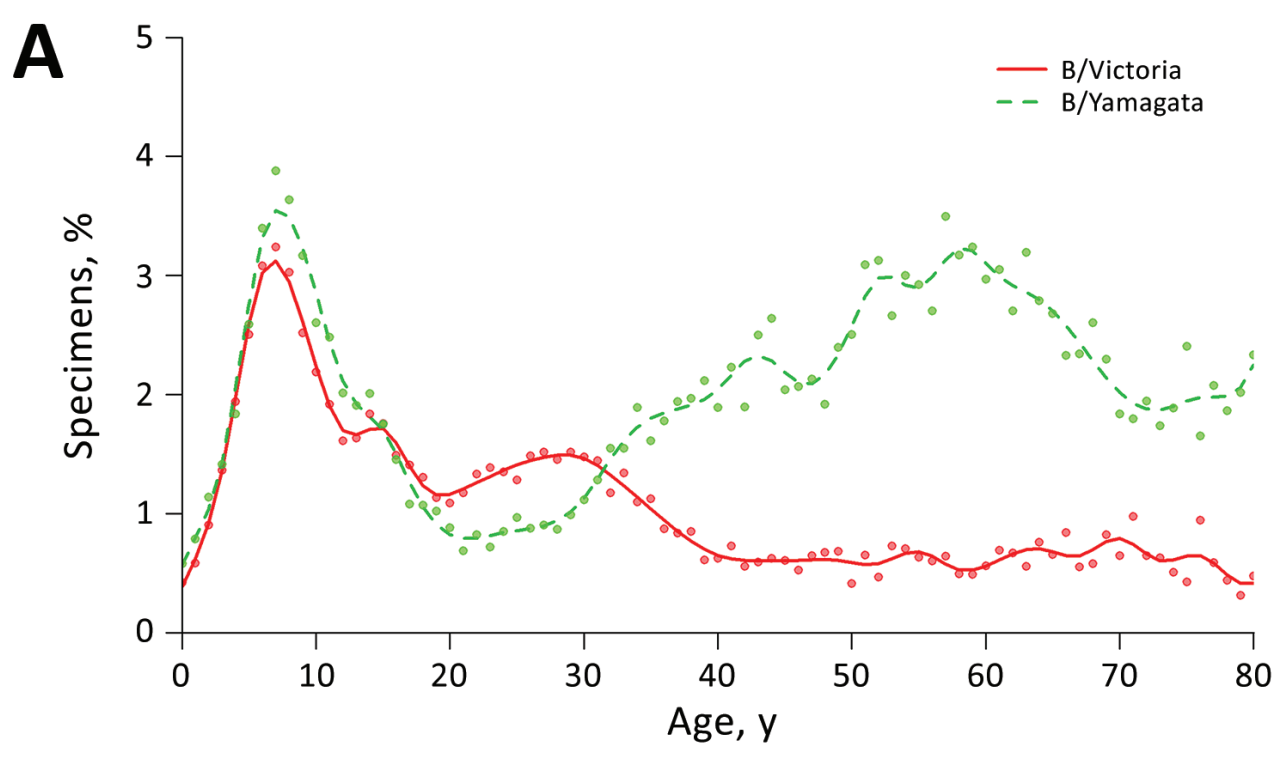

B

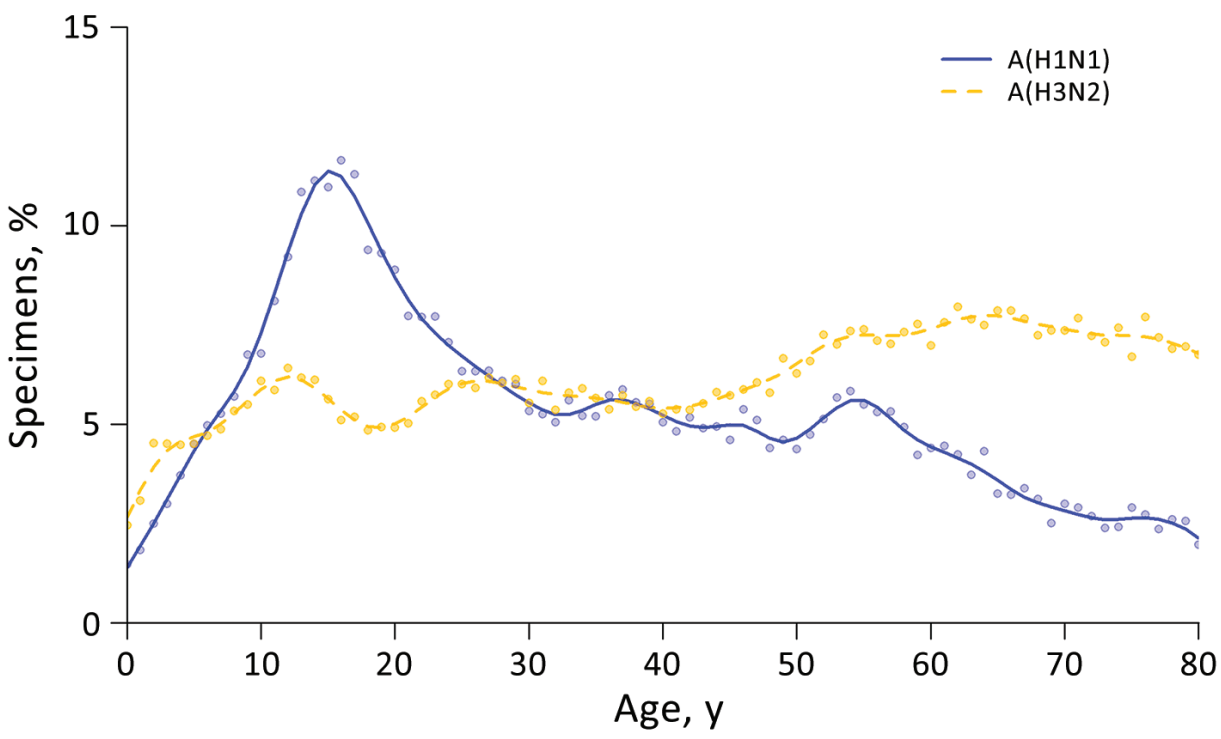

Figure 2. Age-specific proportions of sentinel specimens collected from sentinel surveillance sites testing positive for influenza, China, October 2005-March 2016. A) Influenza B/Victoria and $B /$ Yamagata lineages; $B)$ influenza $A(\mathrm{H} 1 \mathrm{~N} 1)$ and $A(H 3 N 2)$. Findings are based on $2,498,735$ specimens collected from the sentinel hospitals. Dots indicate the original data, and lines (solid and dashed) show the estimation from a fitted smoothing function to the pattern by age. 
B/Victoria (Figure 2). The elevated proportions of infections with both lineages in children might indicate a lack of exposure to the virus early in life $(4,12)$. However, the discrepancy in susceptibility to infections with $\mathrm{B} /$ Victoria and $\mathrm{B} /$ Yamagata in older adults might reflect the genetic difference in viruses of the 2 lineages, although previous exposure to different lineages and vaccination history might have had an effect. Antigenic analysis indicated that circulating $\mathrm{B} / \mathrm{Y}$ amagata strains in general showed a larger genetic diversity than B/Victoria strains (4). This genetic diversity may lead to a substantial number of persons infected with a certain strain of B/Yamagata virus who are susceptible to the other co-circulating strains of the same lineage. The declining frequency of B/ Victoria detections with age, however, implied a gradually strengthened immunity in older persons, which could be attributed to accumulated immunity from exposure to virus strains with fewer genetic changes or possibly to the boosted heterologous immunity against B/Victoria viruses induced by exposure to B/Yamagata viruses (13).

The study has several limitations. First, expansion of the national sentinel surveillance system in China since 2009 might have affected the observed patterns in virus activity because of inclusion of sentinel clinics providing healthcare services specifically to certain populations, such as patients in respiratory or pediatric outpatient clinics, although we weighted virus activity by age in the analysis. Second, the wider application of PCR in national surveillance laboratories might have led to an artificial increase in virus activity; however, we assumed that this change would not differ between the 2 lineages.

Further work could examine the degree of cross-protection conferred by infections of the opposite lineage, if any $(13,14)$. Results from such studies would further elucidate the epidemiology of influenza B virus and optimize vaccination strategies in China.

\section{Acknowledgments}

We thank the staff members at the hospitals; local health departments; and county-, district-, prefecture-, and provinciallevel centers for disease control and prevention for their valuable assistance in coordinating data collection.

This study was funded by the National Science Fund for Distinguished Young Scholars (grant no. 81525023), the US National Institutes of Health (Comprehensive International Program for Research on AIDS grant U19 AI51915), Chinese Center for Disease Control and Prevention's Key Laboratory of Surveillance and Early Warning on Infectious Disease, the Harvard Center for Communicable Disease Dynamics from the National Institute of General Medical Sciences (grant no. U54 GM088558), the Research Grants Council of the Hong Kong Special Administrative Region, China (project no.
T11-705/14N), the National Natural Science Foundation of China (grant no. 81402731), and the Natural Science Foundation of Shanghai (grant no. 14ZR1444500).

Y.H. has received investigator-initiated research funding from Sanofi Pasteur, GlaxoSmithKline, bioMérieux Diagnostic Product (Shanghai), and Yichang HEC Changjiang Pharmaceutical Company. B.J.C. has received research funding from Sanofi Pasteur and consults for Crucell NV.

\section{About the Author}

Dr. Juan Yang was an epidemiologist in the Chinese Center for Disease Control and Prevention, Beijing, China, and currently is an epidemiologist at the School of Public Health, Fudan University, Key Laboratory of Public Health Safety, Ministry of Education, Shanghai, China. Her research interests include epidemiology of infectious diseases, vaccine efficacy/ effectiveness, vaccine policy, and health economics evaluation of vaccination programs to support evidence-based public health decision making.

\section{References}

1. Francis T Jr. A new type of virus from epidemic influenza. Science. 1940;92:405-8. http://dx.doi.org/10.1126/science.92.2392.405

2. Paul Glezen W, Schmier JK, Kuehn CM, Ryan KJ, Oxford J. The burden of influenza B: a structured literature review. Am J Public Health. 2013;103:e43-51. http://dx.doi.org/10.2105/ AJPH.2012.301137

3. Rota PA, Wallis TR, Harmon MW, Rota JS, Kendal AP, Nerome K. Cocirculation of two distinct evolutionary lineages of influenza type B virus since 1983. Virology. 1990;175:59-68. http://dx.doi.org/10.1016/0042-6822(90)90186-U

4. Vijaykrishna D, Holmes EC, Joseph U, Fourment M, Su YC, Halpin R, et al. The contrasting phylodynamics of human influenza B viruses. eLife. 2015;4:e05055. http://dx.doi.org/ 10.7554/eLife.05055

5. Hay AJ, Gregory V, Douglas AR, Lin YP. The evolution of human influenza viruses. Philos Trans R Soc Lond B Biol Sci. 2001;356:1861-70. http://dx.doi.org/10.1098/rstb.2001.0999

6. Tan Y, Guan W, Lam TT, Pan S, Wu S, Zhan Y, et al. Differing epidemiological dynamics of influenza B virus lineages in Guangzhou, southern China, 2009-2010. J Virol. 2013; 87:12447-56. http://dx.doi.org/10.1128/JVI.01039-13

7. Sočan M, Prosenc K, Učakar V, Berginc N. A comparison of the demographic and clinical characteristics of laboratoryconfirmed influenza B Yamagata and Victoria lineage infection. J Clin Virol. 2014;61:156-60. http://dx.doi.org/10.1016/ j.jcv.2014.06.018

8. Shu YL, Fang LQ, de Vlas SJ, Gao Y, Richardus JH, Cao WC. Dual seasonal patterns for influenza, China. Emerg Infect Dis. 2010;16:725-6. http://dx.doi.org/10.3201/ eid1604.091578

9. Wong JY, Wu P, Nishiura H, Goldstein E, Lau EH, Yang L, et al. Infection fatality risk of the pandemic A(H1N1)2009 virus in Hong Kong. Am J Epidemiol. 2013;177:834-40. http://dx.doi.org/10.1093/aje/kws314

10. Caini S, Huang QS, Ciblak MA, Kusznierz G, Owen R, Wangchuk S, et al.; Global Influenza B Study. Epidemiological and virological characteristics of influenza B: results of the Global Influenza B Study. Influenza Other Respi Viruses. 
2015;9(Suppl 1):3-12. http://dx.doi.org/10.1111/ irv.12319

11. Bedford T, Riley S, Barr IG, Broor S, Chadha M, Cox NJ, et al. Global circulation patterns of seasonal influenza viruses vary with antigenic drift. Nature. 2015;523:217-20. http://dx.doi.org/ 10.1038 /nature 14460

12. Peltola V, Ziegler T, Ruuskanen O. Influenza A and B virus infections in children. Clin Infect Dis. 2003;36:299-305. http://dx.doi.org/10.1086/345909

13. Skowronski DM, Hamelin ME, Janjua NZ, De Serres G, Gardy JL, Rhéaume C, et al. Cross-lineage influenza B and heterologous influenza A antibody responses in vaccinated mice: immunologic interactions and $\mathrm{B} /$ Yamagata dominance. PLoS One. 2012;7:e38929. http://dx.doi.org/10.1371/journal.pone.0038929

14. Asahi-Ozaki Y, Yoshikawa T, Iwakura Y, Suzuki Y, Tamura S, Kurata $\mathrm{T}$, et al. Secretory IgA antibodies provide cross-protection against infection with different strains of influenza B virus. J Med Virol. 2004;74:328-35. http://dx.doi.org/10.1002/jmv.20173

Address for correspondence: Hongjie Yu, School of Public Health, Fudan University, Key Laboratory of Public Health Safety, Ministry of Education, 130 Dong'an Rd, Xuhui District, Shanghai 200032, China; email: cfetpyhj@vip.sina.com

\section{The Public Health Image Library (PHIL)}
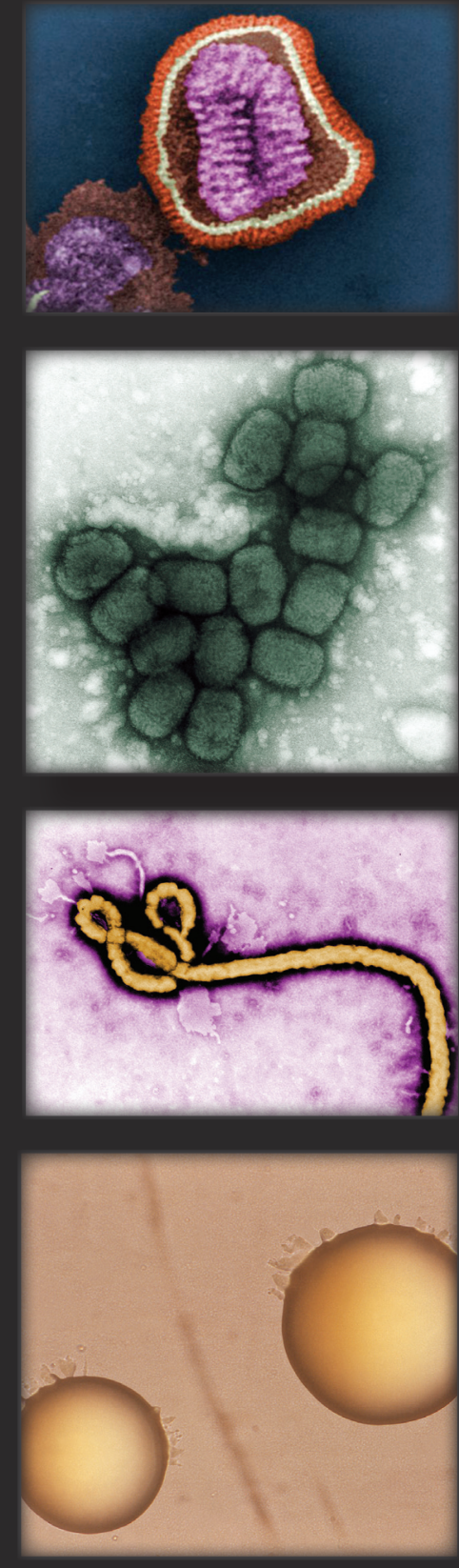

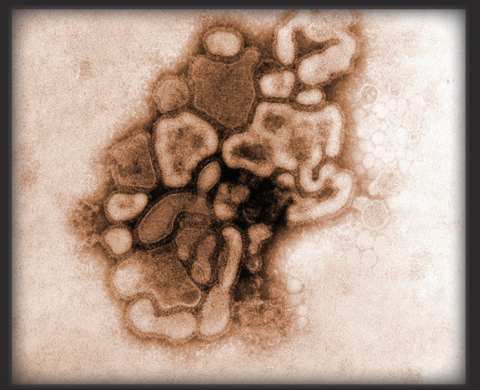

The Public Health Image Library (PHIL), Centers for Disease Control and Prevention, contains thousands of public healthrelated images, including high-resolution (print quality) photographs, illustrations, and videos.

PHIL collections illustrate current events and articles, supply visual content for health promotion brochures, document the effects of disease, and enhance instructional media.

PHIL images, accessible to PC and Macintosh users, are in the public domain and available

without charge.

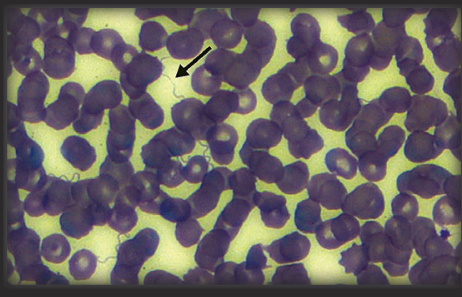

\author{
Visit PHIL at: \\ http: / / phil.cdc.gov/phil
}

